73rd Conference of the Italian Thermal Machines Engineering Association (ATI 2018), 12-14 September 2018, Pisa, Italy

\title{
Vapor-liquid equilibrium of binary systems containing low GWP refrigerants with cubic equations of state
}

\author{
Giovanni Di Nicola ${ }^{\mathrm{a}}$, Gianluca Coccia ${ }^{\mathrm{a}}$, Mariano Pierantozzi ${ }^{\mathrm{b}}$, Sebastiano Tomassettia* \\ ${ }^{a}$ DIISM, Università Politecnica delle Marche, 60131 Ancona, Italy \\ ${ }^{b} S A A D$, Università di Camerino, 63100 Ascoli Piceno, Italy
}

\begin{abstract}
Different Cubic Equations of State (CESs) were used to represent Vapor-Liquid Equilibrium (VLE) of binary systems containing low Global Warming Potential (GWP) refrigerants. The calculated VLE data from some of the widely used CESs were compared with the experimental data collected from the literature. To extend CESs to binary systems, van der Waals one-fluid mixing rules with a single binary interaction parameter were used. Although the deviations between the experimental and calculated values are generally low, a comparison of the results was performed to find the most accurate CES for VLE of the studied binary systems.
\end{abstract}

(C) 2018 The Authors. Published by Elsevier Ltd.

This is an open access article under the CC BY-NC-ND license (https://creativecommons.org/licenses/by-nc-nd/4.0/)

Selection and peer-review under responsibility of the scientific committee of the 73rd Conference of the Italian Thermal Machines

Engineering Association (ATI 2018).

Keywords: HFO; refrigerant binary systems; VLE; cubic equation of state.

\section{Introduction}

The development of accurate models for representation of thermophysical properties and phase equilibria of low Global Warming Potential (GWP) refrigerants, such as HydroFluoro-Olefins (HFOs) and HydroChloroFluoro-Olefins (HCFOs), and their binary systems is of great importance to evaluate the potential performance of working fluids in refrigeration applications. One of the most used and reliable tool to descript the properties of refrigerants is the equation

\footnotetext{
* Corresponding author. Tel: +39 0712204277; fax: +39 071220477110.

E-mail address: s.tomassetti@pm.univpm.it
} 
of state (EOS). Among the many EOSs developed over the years that ranged from simple equations with few constants to complex forms with many constants, the cubic equations of state (CESs) are still models of great interest in many engineering applications. The CESs are semi-empirical expressions derived from the original van der Waals equation of state [1] that are cubic in volume and combine a significant simplicity of calculation with a thermodynamic consistency. In particular, to enhance the representation of the vapor pressure and volumetric properties, a large number of CESs with different expressions and number of parameters were developed [2]. These models have been, and are still, widely used for the representation of the Vapor-Liquid Equilibrium (VLE) of refrigerant mixtures in the academic and industrial fields.

In this work, the VLE of 25 binary systems of alternative refrigerants were descripted using four two-parameters CESs. A wide comparison between the results of the studied CESs were performed on the basis of the experimental data available in literature. The CESs were extended to refrigerant binary systems through van der Waals one-fluid mixing rules with a single binary interaction parameter, also kwon as the standard one-binary-parameter mixing rules. In particular, this parameter was regressed by minimizing the deviations between the calculated and experimental pressure for complete datasets and by minimizing the deviations for each isotherm.

\section{Cubic equations of state}

In general, the two-parameters CESs have the following form:

$$
P=\frac{R \cdot T}{(V-b)}-\frac{a(T)}{\left(V^{2}+u \cdot V+w \cdot b^{2}\right)}
$$

where $P$ is the pressure, $R$ is universal gas constant, $V$ is molar volume, $T$ is temperature, $a(T)$ is temperature dependent parameter, $b$ is parameter not necessarily a function of the temperature, and $u$ and $w$ are two constants that define a specific CES. The parameters $a$ and $b$ in Eq. (1) are:

$$
\begin{aligned}
& a(T)=\Omega_{a} \frac{R^{2} \cdot T_{c}^{2}}{P_{c}} \alpha\left(T_{r}, \omega\right) \\
& b=\Omega_{b} \frac{R \cdot T_{c}}{P_{c}}
\end{aligned}
$$

where $T_{\mathrm{c}}$ is the critical temperature, $P_{\mathrm{c}}$ is the critical pressure, $\alpha\left(T_{\mathrm{r}}, \omega\right)$ is a dimensionless function of the reduced temperature $\left(T_{\mathrm{r}}=T T_{\mathrm{c}}^{-1}\right)$ and acentric factor $(\omega)$, and $\Omega_{a}$ and $\Omega_{b}$ are numerical constants which were calculated from the thermodynamic condition of the critical point. The $\alpha$ function used in this work has the same form of the expression introduced by Soave [3]:

$$
\alpha\left(T_{r}, \omega\right)=\left[1+K \cdot\left(1-T_{r}\right)^{1 / 2}\right]^{2}
$$

where, in this case, $k$ is a function of the acentric factor $(\omega)$; this parameter can be written as: 


$$
k(\omega)=c_{0}+c_{1} \cdot \omega-c_{2} \cdot \omega^{2}+c_{3} \cdot \omega^{3}
$$

where $c_{0}, c_{1}, c_{2}$, and $c_{3}$ are coefficients specific for each CES.

Several two-parameters CESs, characterized by different levels of accuracy, have been proposed in literature by considering different values of $u$ and $w$, including the well-known Redlich-Kwong-Soave (RKS) [3] and PengRobinson (PR) [4] EOSs. Every two-parameters CES is characterized by specific values of constants and critical compressibility factor, $Z_{\mathrm{c}}=P_{\mathrm{c}} V_{\mathrm{c}} R^{-1} T_{\mathrm{c}}^{-1}$. In order to obtain a more accurate description of specific properties for pure fluids and binary systems, many modifications of the proposed CESs were presented in literature. In this respect, the RKS and PR EOSs proposed by Stryjek [5-7], which guarantee a better representation of the saturated vapor pressure of mixture components, shown accurate representation of the vapor-liquid equilibrium (VLE) and critical state for the studied binary systems.

To evaluate the ability of the CESs to represent the VLE for low GWP refrigerant binary systems, the original RKS and PR EOSs were compared together with two CESs proposed by Stryjek [5-7]. The characteristics of the studied CESs (CESs' names, references to the original works and their respective values of $u, w$, coefficients, and $Z_{\mathrm{c}}$ ) are reported in Table 1.

Table 1. Names, references to original work and coefficients of studied CESs.

\begin{tabular}{lllllllllll}
\hline CES Name & Ref. & $u$ & $w$ & $\Omega_{\mathrm{a}}$ & $\Omega_{\mathrm{b}}$ & $Z c$ & $c_{0}$ & $c_{1}$ & $c_{2}$ & $c_{3}$ \\
\hline RKS & {$[3]$} & 1 & 0 & 0.4275 & 0.0866 & 0.333 & 0.4800 & 1.5740 & 0.1760 & 0.0000 \\
PR & {$[4]$} & 2 & -1 & 0.4572 & 0.0778 & 0.307 & 0.3746 & 1.5423 & 0.2699 & 0.0000 \\
PR mod & {$[6,7]$} & 2 & -1 & 0.4572 & 0.0778 & 0.307 & 0.3788 & 1.4895 & 0.1709 & 0.0194 \\
Eq. 1 & {$[6,7]$} & 1 & -1 & 0.4638 & 0.1074 & 0.333 & 0.3577 & 1.4713 & 0.1665 & 0.0183 \\
\hline
\end{tabular}

The standard one-binary-parameter mixing rules, adopted to extend the selected CESs to refrigerant binary systems, are expressed as:

$$
\begin{aligned}
& a_{m}=\sum_{i=1}^{N c} \sum_{j=1}^{N c} y_{i} \cdot y_{j} \cdot a_{i j} \\
& b_{m}=\sum_{i=1}^{N c} y_{i} \cdot b_{i} \\
& a_{i j}=\left(a_{i} \cdot a_{j}\right)^{1 / 2}\left(1-k_{i j}\right)
\end{aligned}
$$

where $N c$ is the number of compounds, $k_{\mathrm{ij}}$ is the binary interaction parameter, $k_{\mathrm{ij}}=k_{\mathrm{ij}}$, and $k_{\mathrm{ii}}=k_{\mathrm{jj}}=0$.

\section{Vapor Liquid Equilibrium of binary systems containing low GWP refrigerants}

After collecting reliable experimental data of VLE for low GWP refrigerant binary systems available in literature, the CESs were tested to represent VLE for these binary systems on the basis of the selected data. Since the simple models under analysis are often inaccurate in the description of the properties of systems containing components that were studied above their critical point, the calculation of VLE data for these binary systems were not considered. 
The number of isotherms, the number of points, the references to the original sources and the ranges of temperature for the binary systems under analysis are reported in Table 2.

Table 2. VLE experimental data for low GWP refrigerant binary systems.

\begin{tabular}{llll}
\hline Binary system & $\begin{array}{l}\text { N. isotherms } \\
\text { (N. points) }\end{array}$ & Reference & T range (K) \\
\hline R1233xf+R244bb & $3(30)$ & {$[8]$} & $323.15-303.15$ \\
R1234yf+R1233xf & $3(30)$ & {$[8]$} & $313.15-293.15$ \\
R1234yf+R152a & $5(60)$ & {$[9]$} & $323.15-283.15$ \\
R1234yf+R227ea & $5(50)$ & {$[10]$} & $323.15-283.15$ \\
R1234yf+R244bb & $3(30)$ & {$[11]$} & $313.15-293.15$ \\
R1234yf+R245cb & $4(44)$ & {$[12]$} & $313.15-283.15$ \\
R1234yf+R290 & $5(54)$ & {$[13]$} & $293.15-253.15$ \\
R1234yf+R600a & $5(60)$ & {$[14]$} & $323.15-283.15$ \\
R1234ze(E)+R290 & $4(38)$ & {$[15]$} & $283.15-258.15$ \\
R1234ze(E)+R600a & $4(40)$ & {$[16]$} & $288.15-258.15$ \\
R125+R1234yf & $7(84)$ & {$[17]$} & $333.21-273.15$ \\
R134+R1234ze(E) & $4(40)$ & {$[18]$} & $288.15-258.15$ \\
R134+R1234ze(Z) & $5(71)$ & {$[19]$} & $343.15-303.15$ \\
R134a+R1234yf & $7(63)$ & {$[17]$} & $333.21-273.31$ \\
R13I1+R1234ze(E) & $5(55)$ & {$[20]$} & $298.15-258.15$ \\
R143a+R1234yf & $5(45)$ & {$[21]$} & $323.15-283.15$ \\
R152a+R1234ze(E) & $4(44)$ & {$[22]$} & $288.15-258.15$ \\
R161+R1234yf & $5(55)$ & {$[23]$} & $323.15-283.15$ \\
& $5(60)$ & {$[24]$} & $323.15-283.15$ \\
R218+R1234yf & $2(20)$ & {$[25]$} & $272.81-223.08$ \\
R23+R1234yf & $3(26)$ & {$[25]$} & $273.25-193.19$ \\
R290+R1234ze(Z) & $5(68)$ & {$[26]$} & $293.15-253.15$ \\
R32+R1234yf & $5(55)$ & {$[23]$} & $323.15-283.15$ \\
& $7(77)$ & {$[17]$} & $333.15-273.15$ \\
R32+R1234ze(E) & $5(15)$ & {$[23]$} & $323.15-283.15$ \\
& $5(65)$ & {$[27]$} & $323.15-283.15$ \\
R600a+R1234ze(Z) & $6(69)$ & {$[28]$} & $353.15-303.15$ \\
R717+R1234yf & $4(58)$ & {$[29]$} & $283.15-253.15$ \\
\hline & & &
\end{tabular}

Table 3 shows the Absolute Average Relative Deviation of pressure (AARD $(\Delta P) \%$ ) and the Absolute Average Deviation of vapor phase mole fraction (AAD $\left(\Delta y_{1}\right)$ ) for the studied binary systems considering $k_{\mathrm{ij}}$ as a constant. In fact, these coefficients were regressed from the complete datasets by minimizing the AARD $(\Delta P) \%$. The AARD of pressure and the AAD of vapor phase mole fraction are respectively defined as:

$$
\begin{aligned}
& A A R D(\Delta P) \%=\frac{100}{N} \sum_{i=1}^{N}\left|\frac{P_{i, \exp }-P_{i, \text { calc }}}{P_{i, \exp }}\right| \\
& A A D(y 1)=\sum_{i=1}^{N}\left|\frac{y_{1 i, \exp }-y_{1 i, \text { calc }}}{N}\right|
\end{aligned}
$$

Table 3. Deviations between experimental and calculated VLE data for binary systems of low GWP refrigerants considering constant $k_{\mathrm{ij}}$.

\begin{tabular}{lllllllll}
\hline Binary systems & RKS & \multicolumn{3}{c}{ PR } & \multicolumn{3}{c}{ PR mod } & Eq. 1 \\
\cline { 2 - 9 } & AARD & AAD & AARD & AAD & AARD & AAD & AARD & AAD \\
& $(\Delta \mathrm{P}) \%$ & $(\Delta \mathrm{y} 1)$ & $(\Delta \mathrm{P}) \%$ & $(\Delta \mathrm{y} 1)$ & $(\Delta \mathrm{P}) \%$ & $(\Delta \mathrm{y} 1)$ & $(\Delta \mathrm{P}) \%$ & $(\Delta \mathrm{y} 1)$ \\
\hline R1233xf+R244bb & 0.9 & 0.0105 & 0.96 & 0.0102 & 1 & 0.0103 & 1.03 & 0.0102 \\
R1234yf+R1233xf & 1.16 & 0.0024 & 1.36 & 0.0012 & 1.37 & 0.0009 & 1.42 & 0.001
\end{tabular}




\begin{tabular}{lllllllll} 
R1234yf+R152a & 0.62 & 0.0029 & 0.24 & 0.0021 & 0.24 & 0.0019 & 0.2 & 0.0021 \\
R1234yf+R227ea & 0.8 & 0.0049 & 0.36 & 0.0034 & 0.4 & 0.0035 & 0.33 & 0.0034 \\
R1234yf+R244bb & 0.83 & 0.0028 & 0.37 & 0.0031 & 0.44 & 0.0033 & 0.38 & 0.0036 \\
R1234yf+R245cb & 0.8 & 0.0106 & 0.38 & 0.0118 & 0.38 & 0.0116 & 0.32 & 0.0119 \\
R1234yf+R290 & 0.29 & 0.0016 & 0.19 & 0.0027 & 0.22 & 0.002 & 0.26 & 0.0024 \\
R1234yf+R600a & 1.01 & 0.0063 & 0.57 & 0.0052 & 0.61 & 0.0048 & 0.53 & 0.0049 \\
R1234ze(E)+R290 & 0.34 & 0.0039 & 0.34 & 0.0041 & 0.32 & 0.0037 & 0.34 & 0.0036 \\
R1234ze(E)+R600a & 0.42 & 0.004 & 0.38 & 0.0028 & 0.43 & 0.003 & 0.49 & 0.0027 \\
R125+R1234yf & 0.72 & 0.012 & 0.4 & 0.0107 & 0.37 & 0.011 & 0.35 & 0.0108 \\
R134+R1234ze(E) & 0.37 & 0.0044 & 0.15 & 0.0032 & 0.18 & 0.0041 & 0.26 & 0.0041 \\
R134+R1234ze(Z) & 3.58 & 0.0138 & 3.41 & 0.0134 & 3.37 & 0.0135 & 3.37 & 0.0135 \\
R134a+R1234yf & 0.81 & 0.0056 & 0.38 & 0.0037 & 0.34 & 0.0039 & 0.28 & 0.0036 \\
R13I1+R1234ze(E) & 0.53 & 0.004 & 0.27 & 0.0044 & 0.21 & 0.0038 & 0.23 & 0.0039 \\
R143a+R1234yf & 0.81 & 0.0027 & 0.4 & 0.0015 & 0.41 & 0.0014 & 0.34 & 0.0017 \\
R152a+R1234ze(E) & 0.64 & 0.0025 & 0.44 & 0.0017 & 0.47 & 0.0023 & 0.52 & 0.0023 \\
R161+R1234yf & 0.84 & 0.0049 & 0.37 & 0.0023 & 0.41 & 0.0025 & 0.33 & 0.0022 \\
& 0.84 & 0.0047 & 0.45 & 0.0036 & 0.5 & 0.0037 & 0.44 & 0.0036 \\
R218+R1234yf & 0.72 & 0.0051 & 0.59 & 0.0049 & 0.69 & 0.0055 & 0.85 & 0.0056 \\
R23+R1234yf & 1.94 & 0.0028 & 1.06 & 0.0029 & 1.19 & 0.0034 & 1.18 & 0.0037 \\
R290+R1234ze(Z) & 1.09 & 0.0071 & 1.24 & 0.008 & 1.15 & 0.008 & 1.18 & 0.008 \\
R32+R1234yf & 0.89 & 0.0057 & 0.63 & 0.0038 & 0.65 & 0.004 & 0.61 & 0.0038 \\
& 0.82 & 0.0098 & 0.51 & 0.0091 & 0.54 & 0.0093 & 0.48 & 0.0092 \\
R32+R1234ze(E) & 0.73 & 0.0051 & 0.67 & 0.0032 & 0.67 & 0.0034 & 0.66 & 0.0031 \\
R600a+R1234ze(Z) & 0.85 & 0.0055 & 0.59 & 0.0052 & 0.6 & 0.0051 & 0.58 & 0.005 \\
R717+R1234yf & 4.37 & 0.0077 & 0.57 & 0.0095 & 0.55 & 0.0092 & 0.58 & 0.0096 \\
Average & 1.06 & 0.00719 & 4.19 & 0.0415 & 3.99 & 0.0415 & 3.93 & 0.0413 \\
\hline
\end{tabular}

The AARD $(\Delta P) \%$ and $\operatorname{AAD}\left(\Delta y_{1}\right)$ for the selected binary systems calculated adopting a temperature dependent $k_{i j}(T)$ for the standard one-binary-parameter mixing rules are reported in Table 4 . In this case, $k_{\mathrm{ij}}$ were regressed by minimizing the AARD $(\Delta P) \%$ for each isotherm.

Table 4. Deviations between experimental and calculated VLE data for binary systems of low GWP refrigerants considering a temperature dependent $k_{\mathrm{ij}}(T)$.

\begin{tabular}{lllllllll}
\hline Binary systems & RKS & \multicolumn{3}{c}{ PR } & \multicolumn{3}{c}{ PR mod } & Eq. 1 \\
\cline { 2 - 9 } & AARD & AAD & AARD & AAD & AARD & AAD & AARD & AAD \\
& $(\Delta P) \%$ & $\left(\Delta y_{1}\right)$ & $(\Delta P) \%$ & $\left(\Delta y_{1}\right)$ & $(\Delta P) \%$ & $\left(\Delta y_{1}\right)$ & $(\Delta P) \%$ & $\left(\Delta y_{1}\right)$ \\
\hline R1233xf+R244bb & 0.80 & 0.0108 & 0.80 & 0.0108 & 0.80 & 0.0108 & 0.80 & 0.0107 \\
R1234yf+R1233xf & 0.65 & 0.0031 & 0.80 & 0.0029 & 0.80 & 0.0025 & 0.85 & 0.0026 \\
R1234yf+R152a & 0.56 & 0.0029 & 0.23 & 0.0022 & 0.24 & 0.0019 & 0.20 & 0.0021 \\
R1234yf+R227ea & 0.74 & 0.0049 & 0.34 & 0.0035 & 0.38 & 0.0035 & 0.32 & 0.0034 \\
R1234yf+R244bb & 0.60 & 0.0026 & 0.22 & 0.0030 & 0.30 & 0.0032 & 0.29 & 0.0034 \\
R1234yf+R245cb & 0.59 & 0.0109 & 0.22 & 0.0120 & 0.24 & 0.0119 & 0.20 & 0.0121 \\
R1234yf+R290 & 0.23 & 0.0018 & 0.14 & 0.0025 & 0.14 & 0.0020 & 0.14 & 0.0023 \\
R1234yf+R600a & 0.99 & 0.0063 & 0.56 & 0.0052 & 0.60 & 0.0049 & 0.52 & 0.0049 \\
R1234ze(E)+R290 & 0.31 & 0.0040 & 0.33 & 0.0043 & 0.31 & 0.0038 & 0.33 & 0.0039 \\
R1234ze(E)+R600a & 0.40 & 0.0041 & 0.28 & 0.0030 & 0.34 & 0.0032 & 0.34 & 0.0031 \\
R125+R1234yf & 0.60 & 0.0116 & 0.33 & 0.0104 & 0.33 & 0.0108 & 0.31 & 0.0106 \\
R134+R1234ze(E) & 0.18 & 0.0039 & 0.12 & 0.0033 & 0.14 & 0.0041 & 0.16 & 0.0041 \\
R134+R1234ze(Z) & 0.45 & 0.0055 & 0.43 & 0.0062 & 0.38 & 0.0059 & 0.45 & 0.0061 \\
R134a+R1234yf & 0.53 & 0.0053 & 0.22 & 0.0037 & 0.20 & 0.0039 & 0.18 & 0.0037 \\
R13I1+R1234ze(E) & 0.34 & 0.0041 & 0.26 & 0.0044 & 0.21 & 0.0038 & 0.20 & 0.0038 \\
R143a+R1234yf & 0.73 & 0.0023 & 0.26 & 0.0017 & 0.30 & 0.0016 & 0.22 & 0.0018 \\
R152a+R1234ze(E) & 0.53 & 0.0025 & 0.41 & 0.0016 & 0.43 & 0.0022 & 0.43 & 0.0022 \\
R161+R1234yf & 0.79 & 0.0048 & 0.33 & 0.0022 & 0.39 & 0.0025 & 0.31 & 0.0021 \\
& 0.79 & 0.0046 & 0.45 & 0.0036 & 0.49 & 0.0037 & 0.44 & 0.0036 \\
R218+R1234yf & 0.66 & 0.0053 & 0.45 & 0.0054 & 0.55 & 0.0056 & 0.62 & 0.0062 \\
R23+R1234yf & 1.62 & 0.0038 & 1.02 & 0.0029 & 1.15 & 0.0033 & 1.17 & 0.0037 \\
R290+R1234ze(Z) & 0.94 & 0.0070 & 1.05 & 0.0081 & 0.95 & 0.0081 & 0.97 & 0.0084 \\
R32+R1234yf & 0.88 & 0.0056 & 0.62 & 0.0039 & 0.64 & 0.0041 & 0.59 & 0.0039 \\
& 0.80 & 0.0097 & 0.51 & 0.0091 & 0.53 & 0.0093 & 0.48 & 0.0092 \\
R32+R1234ze(E) & 0.68 & 0.0050 & 0.60 & 0.0032 & 0.60 & 0.0034 & 0.58 & 0.0031 \\
& 0.84 & 0.0055 & 0.56 & 0.0052 & 0.56 & 0.0051 & 0.54 & 0.0051
\end{tabular}




\begin{tabular}{lllllllll} 
R600a+R1234ze(Z) & 0.54 & 0.0077 & 0.51 & 0.0094 & 0.48 & 0.0090 & 0.50 & 0.0094 \\
R717+R1234yf & 4.24 & 0.0413 & 4.05 & 0.0415 & 3.86 & 0.0411 & 3.80 & 0.0411 \\
Average & 0.80 & 0.0072 & 0.58 & 0.0068 & 0.58 & 0.0068 & 0.57 & 0.0068 \\
\hline
\end{tabular}

Table 3 and Table 4 show that the CESs generally ensure a good representation of the VLE for almost all the studied systems, adopting both constant $k_{\mathrm{ij}}$ and $k_{\mathrm{ij}}(T)$. In the former case, the four CESs gave AARD $(\triangle P) \%$ lower than $2 \%$ and $\operatorname{AAD}\left(\Delta y_{1}\right)$ lower than 0.01 for almost all the studied cases. However, as shown in Table 4, as expected the CESs with $k_{\mathrm{ij}}(T)$ generally produced lower $\operatorname{AARD}(\Delta P) \%$ and $\operatorname{AAD}\left(\Delta y_{1}\right)$ than that of CESs with constant $k_{\mathrm{ij} .}$. As example, the improved description provided by PR EOS with $k_{\mathrm{ij}}$ regressed for each isotherm is shown in Fig. 1 . This figure presents the behaviors of experimental and calculated pressure versus liquid and vapor molar fractions for R134 $+\mathrm{R} 1234 \mathrm{ze}(\mathrm{Z})$ system at $\mathrm{T}=303.15 \mathrm{~K}$ considering both constant $k_{\mathrm{ij}}$ and $k_{\mathrm{ij}}(T)$ for the PR EOS. It is worthwhile noting that, as evident in Fig. 2 for the aforementioned binary system, a slightly variation of $k_{\mathrm{ij}}(T)$ with the temperature guarantees an enhanced representation of the VLE. However, the CESs provided high values of $\operatorname{AARD}(\Delta \mathrm{P}) \%$ and $\operatorname{AAD}(\Delta \mathrm{y} 1)$ for R717 + R1234yf in both the cases.
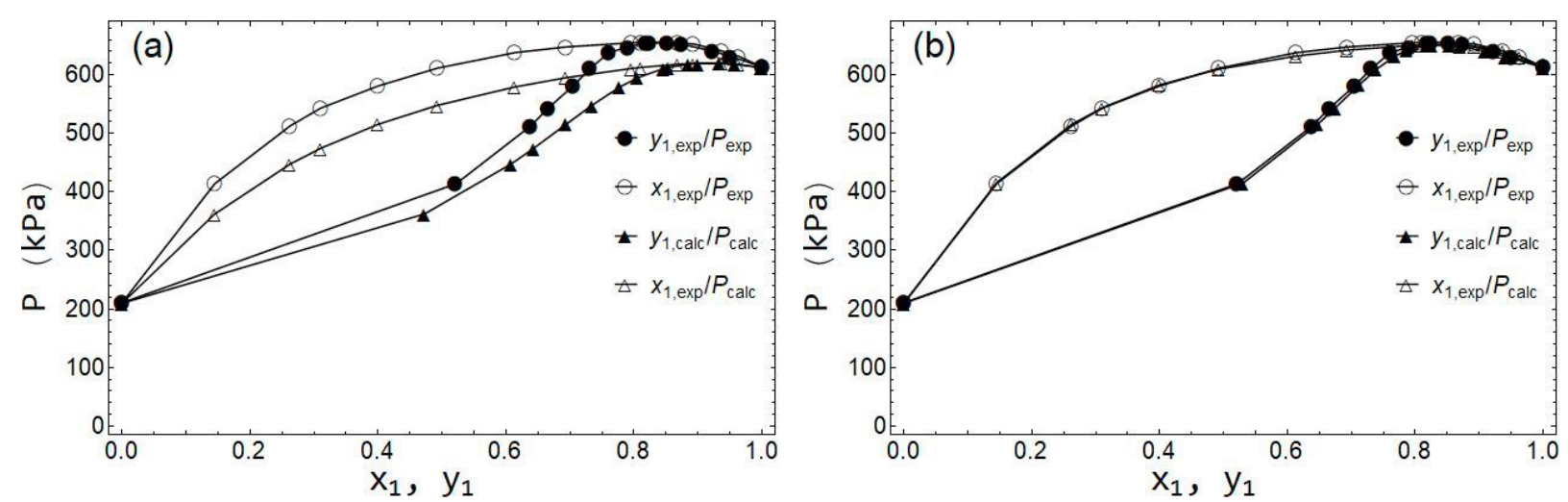

Fig. 1. $P-x-y$ diagram for R134 (1) $+\mathrm{R} 1234 \mathrm{ze}(\mathrm{Z})(2)$ system at T $=303.15 \mathrm{~K}$ considering both (a) constant $k_{\mathrm{ij}}\left(k_{\mathrm{ij}}=0.11116\right)$ and $(\mathrm{b})$ temperature variable $k_{\mathrm{ij}}\left(k_{\mathrm{ij}}=0.14952\right)$ adopting the PR EOS model.

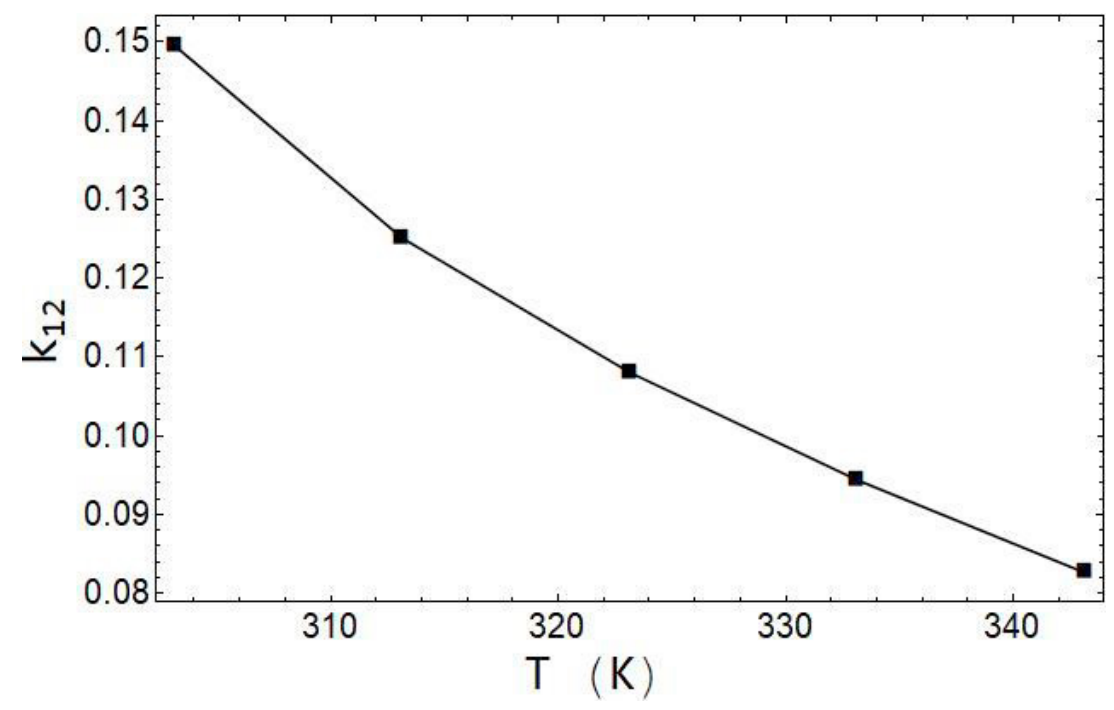

Fig. 2. $k_{\mathrm{ij}}(T)$ as function of the temperature for R134 + R1234ze(Z) adopting the PR EOS model. 


\section{Conclusions}

A literature survey of the experimental VLE data for binary systems of low GWP refrigerants is presented in this work.

These data were studied with the original RKS and PR EOSs. In addition, a modified PR EOS and a CES (Eq.1) proposed by Stryjek were also tested, since they produced lower deviations among the CESs proposed by the same author.

All the models provide satisfactory results for the VLE description, both when the standard one-binary-parameter mixing rules independent on temperature and the same mixing rules dependent on temperature were used.

At the end, the results are generally very good and confirm the representation ability of the CESs, in spite of their simplicity.

\section{Acknowledgements}

This work has been supported by Ministero dell'Istruzione, dell'Università e della Ricerca (MIUR) within the framework of the PRIN 2015 project "Clean Heating and Cooling Technologies for an Energy Efficient Smart Grid".

\section{References}

[1] Van der Waals, Johannes Diderik. Over de Continuiteit van den Gas-en Vloeistoftoestand. Vol. 1. Sijthoff, 1873.

[2] Kayser, R. F., and C. J. Peters. Equations of state for fluids and fluid mixtures. Edited by Jan V. Sengers, and H. J. White. Vol. 5. Amsterdam: Elsevier, 2000.

[3] Soave, Giorgio. "Equilibrium constants from a modified Redlich-Kwong equation of state." Chemical Engineering Science 27, no. 6 (1972): 1197-1203.

[4] Peng, Ding-Yu, and Donald B. Robinson. "A new two-constant equation of state." Industrial \& Engineering Chemistry Fundamentals 15, no. 1 (1976): 59-64.

[5] Stryjek, R. "Correlation and evaluation of VLE data for light n-alkane binary mixtures." Pure and Applied Chemistry 61, no. 8 (1989): 14191427.

[6] Stryjek, R. "CORRELATION AND CRITICAL-EVALUATION OF VLE DATA FOR NITROGEN+ ARGON, NITROGEN+ METHANE, AND ARGON+ METHANE MIXTURES." BULLETIN OF THE POLISH ACADEMY OF SCIENCES-CHEMISTRY 39, no. 4 (1991): 353361 .

[7] Stryjek, R. "ON THE PREDICTION OF THE GAS-LIQUID CRITICAL LOCUS WITH CUBIC EQUATIONS OF STATE." BULLETIN OF THE POLISH ACADEMY OF SCIENCES-CHEMISTRY 40, no. 3 (1992): 211-220.

[8] Yang, Zhi-qiang, Lian-gang Kou, Jing Lu, Wei Zhang, Wei Mao, and Jian Lu. "Isothermal vapor-liquid equilibria measurements for binary systems of 2, 3, 3, 3-tetrafluoropropene (HFO-1234yf)+ 2-chloro-3, 3, 3-trifluoropropene (HCFO-1233xf) and 2-chloro-3, 3, 3-trifluoropropene (HCFO-1233xf)+ 2-chloro-1, 1, 1, 2-tetrafluoropropane (HCFC-244bb)." Fluid Phase Equilibria 414 (2016): 143-148.

[9] Hu, Peng, Long-Xiang Chen, Wan-Bao Zhu, Lei Jia, and Ze-Shao Chen. "Isothermal VLE measurements for the binary mixture of 2, 3, 3, 3tetrafluoroprop-1-ene (HFO-1234yf)+ 1, 1-difluoroethane (HFC-152a)." Fluid Phase Equilibria 373 (2014): 80-83.

[10] Hu, Peng, Long-Xiang Chen, Wan-Bao Zhu, Lei Jia, and Ze-Shao Chen. "Vapor- liquid equilibria for the binary system of 2, 3, 3, 3tetrafluoroprop-1-ene (HFO-1234yf)+ 1, 1, 1, 2, 3, 3, 3-heptafluoropropane (HFC-227ea)." Fluid Phase Equilibria 379 (2014): 59-61.

[11] Yang, Zhi-qiang, Lian-gang Kou, Sheng Han, Chen Li, Zhi-jun Hao, Wei Mao, Wei Zhang, and Jian Lu. "Vapor-liquid equilibria of 2, 3, 3, 3-tetrafluoropropene (HFO-1234yf)+ 1, 1, 1, 2, 2-pentafluoropropane (HFC-245cb) system." Fluid Phase Equilibria 427 (2016): $390-393$.

[12] Yang, Zhi-qiang, Lian-gang Kou, Wei Mao, Jing Lu, Wei Zhang, and Jian Lu. "Isothermal Vapor-Liquid Equilibrium for the Binary System of 2, 3, 3, 3-Tetrafluoropropene and 2-Chloro-1, 1, 1, 2-tetrafluoropropane." Journal of Chemical \& Engineering Data 60, no. 4 (2015): 11531156.

[13] Zhong, Quan, Xueqiang Dong, Yanxing Zhao, Huiya Li, Haiyang Zhang, Hao Guo, and Maoqiong Gong. "Measurements of isothermal vapour-liquid equilibrium for the 2, 3, 3, 3-tetrafluoroprop-1-ene+ propane system at temperatures from 253.150 to $293.150 \mathrm{~K}$." International Journal of Refrigeration 81 (2017): 26-32.

[14] Hu, Peng, Long-Xiang Chen, and Ze-Shao Chen. "Vapor-liquid equilibria for binary system of 2, 3, 3, 3-tetrafluoroprop-1-ene (HFO-1234yf)+ isobutane (HC-600a)." Fluid Phase Equilibria 365 (2014): 1-4.

[15] Dong, Xueqiang, Maoqiong Gong, Jun Shen, and Jianfeng Wu. "Experimental measurement of vapor-liquid equilibrium for (trans-1, 3, 3, 3- 
tetrafluoropropene (R1234ze (E))+ propane (R290))." International journal of refrigeration 34, no. 5 (2011): 1238-1243.

[16] Dong, Xueqiang, Maoqiong Gong, Jun Shen, and Jianfeng Wu. "Vapor-liquid equilibria of the trans-1, 3, 3, 3-tetrafluoropropene (R1234ze (E))+ isobutane (R600a) system at various temperatures from (258.150 to 288.150) K." Journal of Chemical \& Engineering Data 57, no. 2 (2011): 541-544

[17] Kamiaka, Takumi, Chaobin Dang, and Eiji Hihara. "Vapor-liquid equilibrium measurements for binary mixtures of R1234yf with R32, R125, and R134a." International Journal of Refrigeration 36, no. 3 (2013): 965-971.

[18] Dong, Xueqiang, Hao Guo, Maoqiong Gong, Zhi Yang, and Jianfeng Wu. "Measurements of isothermal (vapour+ liquid) equilibria data for $\{1,1,2,2$-Tetrafluoroethane (R134)+ trans-1,3, 3, 3-tetrafluoropropene (R1234ze (E)) $\}$ at T=(258.150 to 288.150) K." The Journal of Chemical Thermodynamics 60 (2013): 25-28.

[19] Zhang, Xuedong, Xueqiang Dong, Hao Guo, Yanxing Zhao, Haiyang Zhang, Maoqiong Gong, and Jun Shen. "Measurements of isothermal (vapour+ liquid) equilibrium for the 1, 1, 2, 2-1, 1, 2, 2-tetrafluoroethane (R134)+ cis-1, 3, 3, 3-tetrafluoropropene (R1234ze (Z)) system at temperatures from (303.150 to 343.150) K." The Journal of Chemical Thermodynamics 111 (2017): 20-26.

[20] Guo, Hao, Maoqiong Gong, Xueqiang Dong, and Jianfeng Wu. "(Vapour+ liquid) equilibrium data for the binary system of \{trifluoroiodomethane (R13I1)+ trans-1, 3, 3, 3-tetrafluoropropene (R1234ze (E))\} at various temperatures from (258.150 to 298.150) K." The Journal of Chemical Thermodynamics 47 (2012): 397-401.

[21] Hu, Peng, Long-Xiang Chen, and Ze-Shao Chen. "Vapor-liquid equilibria for the 1, 1, 1, 2-tetrafluoroethane (HFC-134a)+ 1, 1, 1, 2, 3, 3, 3heptafluoropropane (HFC-227ea) and 1, 1, 1-trifluoroethane (HFC-143a)+2, 3, 3, 3-tetrafluoroprop-1-ene (HFO-1234yf) systems." Fluid Phase Equilibria 360 (2013): 293-297.

[22] Yang, Zhi, Maoqiong Gong, Hao Guo, Xueqiang Dong, and Jianfeng Wu. "Phase equilibrium for the binary mixture of $\{1,1$-difluoroethane (R152a)+ trans-1, 3, 3, 3-tetrafluoropropene (R1234ze (E)) $\}$ at various temperatures from 258.150 to 288.150 K." Fluid Phase Equilibria 355 (2013): 99-103.

[23] Hu, Xiaozhen, Tao Yang, Xianyang Meng, Shengshan Bi, and Jiangtao Wu. "Vapor liquid equilibrium measurements for difluoromethane (R32)+2, 3, 3, 3-tetrafluoroprop-1-ene (R1234yf) and fluoroethane (R161)+2, 3, 3, 3-tetrafluoroprop-1-ene (R1234yf)." Fluid Phase Equilibria 438 (2017): 10-17.

[24] Chen, Long-Xiang, Peng Hu, Wan-Bao Zhu, Lei Jia, and Ze-Shao Chen. "Vapor-liquid equilibria of fluoroethane (HFC-161)+ 2, 3, 3, 3tetrafluoroprop-1-ene (HFO-1234yf)." Fluid Phase Equilibria 392 (2015): 19-23.

[25] Kochenburger, T. M., D. Gomse, I. Tratschitt, A. Zimmermann, and S. Grohmann. "Vapor-liquid and vapor-liquid-liquid equilibrium measurements and correlation of the binary mixtures 2, 3, 3, 3-tetrafluoroprop-1-ene (R1234yf)+(tetrafluoromethane (R14), trifluoromethane (R23), octafluoropropane (R218), nitrogen (R728) and argon (R740)) and ethane (R170)+ trifluoromethane (R23)." Fluid Phase Equilibria 450 (2017): 13-23.

[26] Gong, Maoqiong, Yanxing Zhao, Xueqiang Dong, Hao Guo, Jun Shen, and Jianfeng Wu. "Measurements of isothermal (vapor+ liquid) equilibrium for the (propane+ cis-1, 3, 3, 3-tetrafluoropropene) system at temperatures from (253.150 to 293.150) K." The Journal of Chemical Thermodynamics 98 (2016): 319-323.

[27] Hu, Xiaozhen, Tao Yang, Xianyang Meng, and Jiangtao Wu. "Isothermal vapor liquid equilibrium measurements for difluoromethane (R32)+ fluoroethane (R161)+ trans-1, 3, 3, 3-tetrafluoropropene (R1234ze (E)) ternary mixtures." International Journal of Refrigeration 79 (2017): 49-56.

[28] Zhang, Xuedong, Xueqiang Dong, Hao Guo, Maoqiong Gong, Jun Shen, and Jianfeng Wu. "Measurements and correlations of isothermal (vapour+ liquid) equilibrium for the \{isobutane (R600a) + cis-1, 3, 3, 3-tetrafluoropropene (R1234ze (Z)) $\}$ system at temperatures from (303.150 to 353.150) K." The Journal of Chemical Thermodynamics 103 (2016): 349-354.

[29] Zhao, Yanxing, Xueqiang Dong, Quan Zhong, Huiya Li, Haiyang Zhang, Maoqiong Gong, and Jun Shen. "The experimental investigation of the vapour liquid phase equilibrium for (ammonia+ 2, 3, 3, 3-tetrafluoroprop-1-ene) system." The Journal of Chemical Thermodynamics 113 (2017): 257-262. 